\title{
Simplified Proofs of Error Estimates for the Least Squares Method for Dirichlet's Problem
}

\author{
By Garth A. Baker*
}

\begin{abstract}
Recently, Bramble and Schatz have proposed a projection method for approximating the solution of Dirichlet's problem. Error estimates are derived by the authors using arguments based on certain interpolation theorems for linear operators on Hilbert spaces.

It is shown here that simpler and shorter methods can be used to obtain these error estimates.
\end{abstract}

1. Introduction. The purpose of this note is to give a simplified proof of the results obtained by Bramble and Schatz in [1], where they proposed a method of least squares for obtaining approximations to solutions of Dirichlet's problem.

To obtain error estimates in [1], Bramble and Schatz employed certain interpolation theorems for linear operators on Hilbert space, and, in particular, used these theorems in an iterative argument to get the results.

Subsequently, an observation of Thomée [7] showed that the iterative argument mentioned above could be avoided with the use of appropriate trace theorems and a reformulation of the approximability assumptions on the subspaces used. This observation resulted in a slight simplification of the methods used in [1].

Here, we present a new technique for obtaining the results of [1] which is much simpler and shorter than the prior ones. An entirely different approach is used which in essence involves a basic a priori estimate, and an argument based on duality. This new technique also yields a slight extention of the results of [1]. In particular, the estimate (5.3) of Theorem 5.1 is new.

\section{Notation and Preliminaries.}

Function Spaces. Let $\Omega$ be a bounded domain in $\mathbf{R}^{N}$, with boundary $\partial \Omega$, which is assumed, for convenience, to be of class $C^{\infty}$. The closure of $\Omega$ will be denoted by $\bar{\Omega}$. All functions appearing in this paper will be real valued.

For $s \geqq 0, H^{s}(\Omega)$ and $H^{s}(\partial \Omega)$ will denote the Sobolev spaces of order $s$ of functions on $\Omega$ and $\partial \Omega$, respectively, with associated norms $\|\cdot\|_{s}$ and $|\cdot|_{s}$, respectively. For definitions and characterizations of these spaces, the conventions of [5] are adopted.

On the spaces of square integrable functions on $\Omega$ and $\partial \Omega$, respectively, we shall use the notation

Received September 4, 1972.

AMS (MOS) subject classifications (1970). Primary 65N30.

* Sponsored by the United States Army under Contract No. DA-31-124-ARO-D-462.

Copyright @ 1973, American Mathematical Society 


$$
(u, v)=\int_{\Omega} u v d x \text { and }\langle u, v\rangle=\int_{\partial \Omega} u v d \sigma
$$

for the inner products.

For $s<0$, the conventions of [6] are adopted; $H^{s}(\Omega)$ is defined to be the completion of $C^{\infty}(\bar{\Omega})$, the set of infinitely differentiable functions on $\bar{\Omega}$, with respect to the norm

$$
\|v\|_{s}=\sup _{\psi \in C^{\infty}(\bar{\Omega})}(v, \psi) /\|\psi\|_{-s} .
$$

Similarly, by the conventions of [6], for $s<0, H^{s}(\partial \Omega)$ is defined to be the completion of $C^{\infty}(\partial \Omega)$, the set of infinitely differentiable functions on $\partial \Omega$, with respect to the norm

$$
|v|_{s}=\sup _{\psi \in C^{\infty}(\partial \Omega)}\langle v, \psi\rangle /|\psi|_{-s} .
$$

The Boundary-Value Problem. As in [1], we are concerned with the method of least squares for obtaining approximations to the solution, $u$, of the elliptic boundaryvalue problem

$$
\begin{array}{cc}
L u=f & \text { in } \Omega, \\
u=g & \text { on } \partial \Omega .
\end{array}
$$

Here, $L$ is the second-order elliptic operator defined by

$$
L u=L(x, D) u=-\sum_{i, j=1}^{N} D_{i}\left(a_{i j}(x) D_{i} u\right)+\sum_{i=1}^{N} b_{i}(x) D_{i} u+c(x) u, \quad x \in \Omega,
$$

$f \in H^{0}(\Omega)$ and $g \in H^{3 / 2}(\partial \Omega)$. This problem is denoted by $\odot(L, f, g)$.

The following condition is imposed on the problem:

Condition (A). (i) The coefficients $a_{i j}, b_{i}$ and $c$ of $L$ are in $C^{\infty}(\bar{\Omega}), i, j=1,2, \cdots, N$.

(ii) $L$ is uniformly elliptic in $\bar{\Omega}$. That is, there exists a constant $\alpha>0$, such that, for $\left(\xi_{1}, \xi_{2}, \cdots, \xi_{N}\right) \in \mathbf{R}^{N}$ and all $x \in \bar{\Omega}$,

$$
\alpha \sum_{i=1}^{N} \xi_{i}^{2} \leqq \sum_{i, j=1}^{N} a_{i j}(x) \xi_{i} \xi_{i}
$$

(iii) The only solution of $\mathcal{P}(L, 0,0)$ is $u=0$.

$A$ Priori Estimates. In this section and throughout the rest of the paper, $C$ will denote a generic constant, not necessarily the same in any two places.

Concerning the existence, uniqueness, and regularity of the solution of (2.1), we have the following theorem.

THEOREM 2.1 (CF. [5]). Under Condition (A), the mapping $T: C^{\infty}(\Omega) \rightarrow C^{\infty}(\Omega) \times$ $C^{\infty}(\partial \Omega)$ defined by $T u=(L u, u)$ and completed by continuity is a homeomorphism of $H^{s}(\Omega)$ onto $H^{s-2}(\Omega) \times H^{s-1 / 2}(\partial \Omega)$ for all $s \geqq 2$; and the norms

$$
\|\cdot\|_{s} \text { and }\left\{\|L \cdot\|_{s-2}^{2}+|\cdot|_{s-1 / 2}^{2}\right\}^{1 / 2}
$$

are equivalent.

Henceforth, we assume that the boundary-value problem (2.1) has a unique solution $u \in H^{r}(\Omega)$, for some $r \geqq 4$.

We shall need also the following a priori estimates.

TheOREM 2.2 (CF. [6]). Under Condition (A), for each $s \geqq 2$, if $v \in H^{s}(\Omega)$, then 
$L v \in H^{s-2}(\Omega)$, and $v \in H^{s-1 / 2}(\partial \Omega)$ and, for each $p \leqq s$, there exists a constant $C_{\nu}$ independent of $v$, such that

$$
\|v\|_{p} \leqq C_{p}\left\{\|L v\|_{p-2}+|v|_{p-1 / 2}\right\} .
$$

Lemma 2.1 (CF. [4]). There exists a constant $C>0$ such that, for all $v \in H^{1}(\Omega)$,

$$
|v|_{0} \leqq C\left\{\epsilon^{-1}\|v\|_{0}+\epsilon\|v\|_{1}\right\}
$$

for all $\epsilon>0$.

LEMMA 2.2 (CF. [5]). There exists a constant $C$ such that, if $s_{1}<s_{2}$ are any two real numbers, then, for $s=\theta s_{1}+(1-\theta) s_{2}, 0 \leqq \theta \leqq 1$, and $v \in H^{s_{2}}(\partial \Omega)$,

$$
|v|_{s} \leqq C|v|_{s_{1}}^{\theta}|v|_{s_{2}}^{(1-\theta)} \text {. }
$$

3. Finite-Dimensional Subspaces. Certain classes of finite-dimensional function spaces, from which approximations to the solution of (2.1) are obtained, will now be introduced.

Let $r$ and $k$ be given positive integers with $k \leqq r$. We say that the family of spaces $\left\{S_{h}\right\}_{0<h \leqq 1}$ is of class $S_{k, r}(\Omega)$ if

(a) for each $h, S_{h}$ is finite dimensional,

(b) $S_{h} \subset H^{k}(\Omega)$ for each $h$,

(c) there is a constant $C$ such that if $v \in H^{r}(\Omega)$,

$$
\inf _{x \in S_{h}} \sum_{i=0}^{k} h^{i}\|v-\chi\|_{i} \leqq C h^{r}\|v\|_{r}, \quad 0<h \leqq 1 .
$$

It can be shown that if $\left\{S_{h}\right\}_{0<h \leqq 1}$ is of class $S_{k, r}(\Omega)$ with $2 \leqq k$, then it is also of class $S_{2,8}(\Omega)$ for all $2 \leqq s \leqq r$. This is done in [2].

The approximability assumptions on the subspaces $\left\{S_{h}\right\}_{0<h \leqq 1}$ differ from those of [1]; however, for the types of subspaces used in practice, for example piecewise polynomials, it may be shown that both are satisfied (cf. [3]).

Henceforth, we assume that we have a family of subspaces $\left\{S_{h}\right\}_{0<h \leqq 1}$, of class $\varsigma_{k, r}(\Omega)$, for some $r \geqq 4$, and $k \geqq 2$, both fixed.

4. Least Squares Approximations. On $H^{2}(\Omega) \times H^{2}(\Omega)$, define the bilinear form $[\cdot, \cdot]$ by

$$
[\varphi, \psi]=(L \varphi, L \psi)+h^{-3}\langle\varphi, \psi\rangle
$$

where $h$ is as in Section 3.

Clearly, $[\cdot, \cdot]$ defines an inner product, and we introduce the notation

$$
\|\varphi\|_{L}=[\varphi, \varphi]^{1 / 2} \text {. }
$$

We also have Schwarz's inequality

$$
[\varphi, \psi] \leqq\|\varphi\|_{L}\|\psi\|_{L} .
$$

LeMmA 4.1. Let $u$ satisfy (2.1), then, for each $h \in(0,1]$, there exists a unique function $u_{h} \in S_{h}$ satisfying

$$
\left[u_{h}, \chi\right]=(f, L \chi)+h^{-3}\langle g, \chi\rangle \text { for all } \chi \in S_{h},
$$




$$
\left[u-u_{h}, \chi\right]=0 \quad \text { for all } \chi \in S_{h}
$$

and

$$
\inf _{x \in S_{h}}\|u-\chi\|_{L}=\left\|u-u_{h}\right\|_{L}
$$

Proof. The existence and uniqueness of $u_{h} \in S_{h}$ satisfying (4.2) follows from the fact that $S_{h}$ is finite dimensional, and that the form $[\cdot, \cdot]$ is positive definite.

Now, observe that if $u$ satisfies (2.1), then $[u, \chi]=(f, L \chi)+h^{-3}\langle g, \chi\rangle$ and hence, from (4.2), we have in fact

$$
[u, \chi]=\left[u_{h}, \chi\right] \text { for all } \chi \in S_{h},
$$

which gives (4.3).

Clearly,

$$
\inf _{\chi \in S_{h}}\|u-\chi\|_{L} \leqq\left\|u-u_{h}\right\|_{L}
$$

For $\chi \in S_{h}, \chi-u_{h} \in S_{h}$, and so

$$
\begin{aligned}
\left\|u-u_{h}\right\|_{L}^{2} & =\left[u-u_{h}, u-u_{h}\right]=\left[u-u_{h}, u-\chi+\chi-u_{h}\right] \\
& =\left[u-u_{h}, u-\chi\right] \leqq\left\|u-u_{h}\right\|_{L}\|u-\chi\|_{L}
\end{aligned}
$$

that is

$$
\left\|u-u_{h}\right\|_{L} \leqq\|u-\chi\|_{L}, \quad \chi \in S_{h} .
$$

Hence

$$
\left\|u-u_{h}\right\|_{L} \leqq \inf _{x \in S_{\Lambda}}\|u-\chi\|_{L} .
$$

Combining (4.5) and (4.6), we get (4.4). This concludes the proof.

The function $u_{h}$ in the above lemma is called the least squares approximation to $u$, and (4.4) is thus an error estimate for the approximation. In fact, (4.4) shows that $u_{h}$ is the best approximation to the function $u$, from the subspace $S_{h}$, in the norm $\|\cdot\|_{L}$.

The following lemma now displays the approximability of the family of subspaces $\left\{S_{h}\right\}_{0<h \leqq 1}$ with respect to the norm $\|\cdot\|_{L}$.

LEMMA 4.2. There exists a constant $C>0$ such that, for $2 \leqq s \leqq r$ and $v \in H^{*}(\Omega)$,

$$
\inf _{\chi \in S_{h}}\|v-\chi\|_{L} \leqq C h^{s-2}\|v\|_{s}, \quad 0<h \leqq 1 .
$$

Proof. Let $\chi \in S_{h}$. Then

$$
\begin{aligned}
\|v-\chi\|_{L} & =\left\{\|L(v-\chi)\|_{0}^{2}+h^{-3}|v-\chi|_{0}^{2}\right\}^{1 / 2} \\
& \leqq C\left\{\|v-\chi\|_{2}+h^{-3 / 2}|v-\chi|_{0}\right\},
\end{aligned}
$$

from Theorem 2.1, for some constant $C$. From Lemma 2.1, with $\epsilon=h^{1 / 2}$,

$$
h^{-3 / 2}|v-\chi|_{0} \leqq C h^{-3 / 2}\left\{h^{-1 / 2}\|v-\chi\|_{0}+h^{1 / 2}\|v-\chi\|_{1}\right\} .
$$

Hence, for some constant $C$,

$$
\|v-\chi\|_{L} \leqq C h^{-2} \sum_{j=0}^{2} h^{i}\|v-\chi\|_{i}
$$


And so, from the property (c) of the subspaces of Section 3,

$$
\inf _{x \in S_{h}}\|v-\chi\|_{L} \leqq C h^{s-2}\|v\|_{s},
$$

which concludes the proof.

5. Error Estimates. In this section, estimates for $L\left(u-u_{h}\right)$ and for the error $\left(u-u_{h}\right)$ on the boundary $\partial \Omega$ are derived; these estimates are then combined to give estimates for the error in the spaces $H^{p}(\Omega), 4-r \leqq p \leqq \frac{1}{2}$. The following theorem is a slight extension of the results of Bramble and Schatz. The proofs given here are new, and constitute the main part of the paper.

THEOREM 5.1. There exists a constant $C$ such that, if $u$ satisfies (2.1), and $u_{h} \in S_{h}$ satisfies (4.2), then, for $2 \leqq s \leqq r$, if $u \in H^{s}(\Omega)$ and $e=u-u_{h}$,

$$
\begin{aligned}
\|e\|_{L} & \leqq C h^{8-2}\|u\|_{s}, & \\
\|L e\|_{p-2} & \leqq C h^{s-p}\|u\|_{s}, & 4-r \leqq p \leqq 2, \\
|e|_{p-1 / 2} & \leqq C h^{s-p}\|u\|_{s}, & 1-r \leqq p \leqq \frac{1}{2}, \\
\|e\|_{p} & \leqq C h^{8-p}\|u\|_{s}, & 4-r \leqq p \leqq \frac{1}{2} .
\end{aligned}
$$

Proof. The estimate (5.1) follows immediately from (4.4) of Lemma 4.1 and Lemma 4.2.

We now derive (5.2) and (5.3). To this end, let $\psi \in C^{\infty}(\bar{\Omega})$ and $v \in C^{\infty}(\partial \Omega)$. By Theorem 2.1, there exists a $\varphi \in C^{\infty}(\bar{\Omega})$, satisfying

$$
\begin{array}{cc}
L \varphi=\psi & \text { in } \Omega, \\
\varphi=v & \text { on } \partial \Omega,
\end{array}
$$

hence

$$
[e, \varphi]=(L e, \psi)+h^{-3}\langle e, v\rangle
$$

Now, by (4.3) and (4.1),

$$
[e, \varphi]=[e, \varphi-\chi] \leqq\|e\|_{L}\|\varphi-\chi\|_{L}
$$

for all $\chi \in S_{h}$. Thus, by Lemma 4.2 ,

$$
[e, \varphi] \leqq\|e\|_{L} \inf _{\chi \in S_{h}}\|\varphi-\chi\|_{L} \leqq C h^{s-2}\|\varphi\|_{8}\|e\|_{L}
$$

for $2 \leqq s \leqq r$. Hence, from (2.2),

$$
(L e, \psi)+h^{-3}\langle e, v\rangle \leqq C h^{s-2}\|e\|_{L}\left\{\|\psi\|_{s-2}+|v|_{s-1 / 2}\right\}
$$

for $2 \leqq s \leqq r$.

Now, (5.5) holds for $\psi$ arbitrary in $C^{\infty}(\bar{\Omega})$, and $v$ arbitrary in $C^{\infty}(\partial \Omega)$. Hence, in particular, for $v=0$, we have

$$
(L e, \psi) \leqq C h^{s-2}\|e\|_{L}\|\psi\|_{s-2}, \quad \psi \in C^{\infty}(\bar{\Omega}), 2 \leqq s \leqq r .
$$

Therefore

$$
\|L e\|_{-(s-2)}=\sup _{\psi \in C^{\infty}(\bar{\Omega})}(L e, \psi) /\|\psi\|_{s-2} \leqq C h^{s-2}\|e\|_{L}, \quad 2 \leqq s \leqq r,
$$


or

$$
\|L e\|_{p-2} \leqq C h^{2-p}\|e\|_{L}, \quad 4-r \leqq p \leqq 2 .
$$

Hence, from (5.1) and (5.6), we get

$$
\|L e\|_{D-2} \leqq C h^{s-D}\|u\|_{s}, \quad 4-r \leqq p \leqq 2,
$$

which is (5.2).

Similarly, for $\psi=0$ in (5.5),

$$
\langle e, v\rangle \leqq C h^{s+1}|v|_{s-1 / 2}\|e\|_{L}, \quad v \in C^{\infty}(\partial \Omega), 2 \leqq s \leqq r,
$$

and so

$$
|e|_{-(s-1 / 2)}=\sup _{v \in C^{\infty}(\partial \Omega)}\langle e, v\rangle /|v|_{s-1 / 2} \leqq C h^{s+1}\|e\|_{L}, \quad 2 \leqq s \leqq r .
$$

In particular, for $s=r$ in (5.7), we get

$$
|e|_{-(r-1 / 2)} \leqq C h^{r+1}\|e\|_{L} .
$$

We also have from the definition of the norm $\|\cdot\|_{L}$ that

$$
|e|_{0} \leqq h^{3 / 2}\|e\|_{L} .
$$

Using (5.8) and (5.9), we can estimate $|e|_{p-1 / 2}$ for $1-r \leqq p \leqq \frac{1}{2}$. Precisely, we may write

$$
p-\frac{1}{2}=\theta\left[-\left(r-\frac{1}{2}\right)\right], \quad 0 \leqq \theta \leqq 1 .
$$

And from Lemma 2.2, with $s_{2}=0, s_{1}=-\left(r-\frac{1}{2}\right)$,

(5.10) $\quad|e|_{p-1 / 2} \leqq C|e|_{0}^{(1-\theta)}|e|_{-(r-1 / 2)}^{\theta}$, where $\theta=-\left(p-\frac{1}{2}\right) /\left(r-\frac{1}{2}\right)$.

Hence, using (5.8) and (5.9) in (5.10),

$$
|e|_{p-1 / 2} \leqq C h^{2-p}\|e\|_{L}, \quad 1-r \leqq p \leqq \frac{1}{2} .
$$

And again, from (5.1),

$$
|e|_{p-1 / 2} \leqq C h^{s-p} \mid\|u\|_{s}, \quad 1-r \leqq p \leqq \frac{1}{2},
$$

which is (5.3). (2.2),

We may now obtain (5.4) from (5.2) and (5.3), by using the a priori inequality

$$
\|e\|_{p} \leqq C\left\{\|L e\|_{p-2}+|e|_{p-1 / 2}\right\} .
$$

Combining (5.2) and (5.3), we obtain, for $4-r \leqq p \leqq \frac{1}{2}$,

$$
\|e\|_{p} \leqq C h^{s-p}\|u\|_{s}, \quad 2 \leqq s \leqq r,
$$

which concludes the proof. 
1. J. H. Bramble \& A. H. Schatz, "Rayleigh-Ritz-Galerkin methods for Dirichlet's problem using subspaces without boundary conditions," Comm. Pure Appl. Math., v. 23, 1970, pp. 653-675. MR 42 \#2690.

2. J. H. Bramble, T. Dupont \& V. Thomée, Higher Order Polygonal Domain Galerkin Approximations in Dirichlet's Problem, MRC Technical Summary Report \#1213, March 1972.

3. J. H. Bramble \& S. R. HilberT, "Estimation of linear functionals on Sobolev spaces with application to Fourier transforms and spline interpolation," SIAM J. Numer. Anal., v. 7, 1970, pp. 112-124. MR 41 \#7819.

4. J. H. Bramble \& V. ThOMÉE, "Semidiscrete-least squares methods for a parabolic boundary value problem," Math. Comp., v. 26, 1972, pp. 633-648.

5. J. L. Lions \& E. MAgÈnes, Prob̆lèmes aux Limites non Homogènes et Applications.

Vol. 1, Travaux et Recherches Mathématiques, no. 17, Dunod, Paris, 1968. MR 40 \# 512.

5. M. Schechter, "On $L^{p}$ estimates and regularity. II," Math. Scand., v. 13, 1963, pp. 47-69. MR 32 \#6052.

7. V. THOMÉE, Private communication. 